\title{
Parâmetros eletrocardiográficos de equinos desclassificados por exaustão em competições de enduro
}

[Electrocardiographic parameters of horses disqualified from exhaustion in endurance competitions]

\author{
C.B.S. Dumont ${ }^{1}$, J.M. Moraes $^{2}$, C.R. Leite $^{1}$, R.O. Alves ${ }^{2}$, M. Moreira ${ }^{3}$, \\ A.R.C. Moscardini ${ }^{4}$, R.F. Godoy ${ }^{1}$, E.M.M. Lima ${ }^{1 *}$ \\ ${ }^{1}$ Universidade de Brasília - Brasília DF \\ ${ }^{2}$ Universidade Federal de Goiás - Goiânia GO \\ ${ }^{3} 1^{\circ}$ Regimento de Cavalaria de Guarda - Brasília, DF \\ ${ }^{4}$ Regimento de Polícia Montada PM-DF - Brasília, DF
}

\begin{abstract}
RESUMO
Foram examinados, por meio de eletrocardiografia, 14 equinos Puro Sangue Árabe, 12 machos e duas fêmeas, desclassificados por exaustão em provas de enduro entre 60 e $160 \mathrm{~km}$. Foi observado predomínio de taquicardia sinusal, seguido de arritmia sinusal e complexo atrial prematuro com a frequência cardíaca variando de 48 a 78bpm e 93 a 111bpm, respectivamente. Ocorreu aumento da amplitude das ondas P, R, $S$ e T e redução nas suas durações, bem como redução nos intervalos e segmentos, porém o complexo QRS quase não se alterou. O alongamento observado do QTc indicou fadiga miocárdica moderada em resposta ao exercício, e o supradesnível ST foi indicativo de hipovolemia. O eixo elétrico no plano frontal apresentou desvio à direita, aumento de câmara e hipertrofia secundários ao treinamento.
\end{abstract}

Palavras-chave: equino, eletrocardiograma, enduro, fadiga

\begin{abstract}
By using electrocardiographic examination, 14 Purebred Arabian horses, 12 males and two females, disqualified due to exhaustion in endurance races from 60 and $160 \mathrm{~km}$ were evaluated. Predominance of sinus tachycardia, followed by sinus arrhythmia and atrial premature complex, with the heart rate ranging from 48 to $78 \mathrm{bpm}$, and 93 to $111 \mathrm{bpm}$, respectively, were observed. There was increase in the amplitude of waves $P, R, S$, and $T$, and decrease in their durations, as well as reduction in the intervals and segments; nevertheless, the QRS complex was not almost altered. The QTC lengthening pointed to a moderate myocardial fatigue in response to exercise, and the elevation of ST was indicative of hypovolemia. The electrical axis in frontal plane presented deviation to the right, chamber increase, and hypertrophy secondary to training.
\end{abstract}

Keywords: equine, electrocardiography, endurance, fatigue

\section{INTRODUÇÃO}

Não existem métodos fáceis para avaliar o desempenho atlético de um cavalo. Dentre os possíveis, destacam-se os que envolvem a esfera da fisiologia do exercício (Hinchclif e Geor, 2004; Boffi, 2006), o manejo nutricional específico, a atividade realizada pelo animal (Peter, 2003), a avaliação do estresse oxidativo
(Gondim et al., 2009), a fisiologia respiratória (Petsche et al., 1995) e os dados relativos às propriedades musculoesqueléticas (Serrano et al., 2000). Da mesma forma, avaliam-se os eletrólitos, a glicose e a bioquímica sanguínea (Fernandes e Larsson, 2000) e ainda a condição cardíaca (Fazio et al., 2003; Fernandes et al., 2004). Desse modo, pode-se estabelecer um programa de treinamento adequado que resulte

Recebido em 5 de abril de 2010

Aceito em 22 de dezembro de 2010

*Autor para correspondência (corresponding author)

E-mail: limaemm@unb.br 
na expressão máxima do potencial atlético do indivíduo (Boffi, 2006).

Wickler e Foss (2004) e Boffi (2006) definiram como exaustão a síndrome metabólica resultante dos efeitos deletérios decorrentes da desidratação, de distúrbios eletrolíticos, da hipertermia e da depleção dos substratos energéticos (metabolismo oxidativo). Animais afetados podem apresentar depressão, fraqueza, tropeços à deambulação, claudicação, anorexia, olhar vidrado sem expressão e mucosas secas e congestas (Wickler e Foss, 2004; Boffi, 2006).

Para Muriel (2006), o sistema cardiovascular de equinos atletas desempenha importante papel na manutenção da volemia, ao promover aporte sanguíneo extra e manutenção da frequência cardíaca durante o exercício. Isto sugere que a avaliação deste sob efeito de exercício específico permite não só estimar a influência de anomalias cardíacas, mas também os efeitos deletérios do esforço realizado frente à função cardíaca (Evans, 1994).

Babusci e López (2006) comentaram que em cavalos desidratados e com transtornos eletrolíticos graves, ocorreram alterações no potencial da membrana e que estes apresentaram taquicardia ventricular, com influência direta no desempenho atlético. Sugeriram, ainda, que o exame eletrocardiográfico após o exercício poderia melhor avaliar a função cardíaca, detectando arritmias que não seriam encontradas em repouso, assim como o aumento de câmaras, hipertrofias e dilatações. Heidbuchel et al. (2003) verificaram que esportes de resistência estariam relacionados à ocorrência e progressão de arritmias ventriculares, sendo estas consideradas como benignas ou não, portanto avaliar o risco de evolução e a origem do evento arrítmico seria de grande importância.

Dessa forma, o presente trabalho teve como objetivo verificar o comportamento da função cardíaca de equinos Puro Sangue Árabe desclassificados por exaustão, em provas de enduro de longa duração, por meio da avaliação dos parâmetros eletrocardiográficos.

\section{MATERIAL E MÉTODOS}

Foram examinados 14 equinos desclassificados por exaustão, sendo 12 machos e duas fêmeas, participantes de campeonato regional da Federação Hípica de Brasília no Distrito Federal, quando percorreram de 60 a $160 \mathrm{~km}$, caracterizando exercício de baixa intensidade e longa duração, com velocidade média de $20 \mathrm{~km} / \mathrm{h}$. Os desclassificados por exaustão foram aqueles acometidos por distúrbios metabólicos com comprometimento de suas funções vitais (Wickler e Foss, 2004), ressaltando-se que os animais claudicantes não foram avaliados. O estudo foi submetido e obedeceu às normas do Comitê de Ética no Uso Animal da Universidade de Brasília sob protocolo n ${ }^{\circ}$ 88-2009.

Os traçados eletrocardiográficos foram obtidos durante 60 segundos utilizando-se o aparelho C10 TEB, sistema composto por eletrocardiógrafo digital de 12 canais e software ECGPC Veterinário versão 2,27 compatível com ambiente Windows, em velocidade de registro dos traçados de $25 \mathrm{~mm} / \mathrm{s}$ com ajuste da sensibilidade do aparelho para $1 \mathrm{mV}=1 \mathrm{~cm}$. Foram registradas as derivações bipolares I, II, III e unipolares amplificadas, aVR, aVF, aVL. A avaliação ocorreu imediatamente após a desclassificação do animal pela equipe veterinária oficial da competição.

Os cavalos foram avaliados no momento da desclassificação, isto é, após inspeção veterinária oficial (vetcheck), quando os animais foram contidos fisicamente em posição ortostática, com os membros paralelos entre si, todos com ferradura, sem qualquer tranquilização, e antes da realização de qualquer tipo de tratamento. Foi aferida ainda a temperatura retal (TR) e a mensuração do peso corpóreo por meio de fita de peso, além da coleta de amostras de sangue venoso para avaliação hematológica e hemogasométrica.

A disposição dos eletrodos seguiu o sistema de derivação bipolar ápice-base, descrito por Dumont et al. (2010). Tais eletrodos estavam presos à pele por meio de condutores metálicos (clipe tipo jacaré) e embebidos com álcool. Os dados foram gravados em ambiente Windows mediante o uso do software empregado.

Os parâmetros eletrocardiográficos foram avaliados por meio de análise sistemática, compreendendo a medida em milivolts das amplitudes das ondas e segmentos, e em milissegundos, a duração das ondas, dos 
intervalos e complexos, bem como foi promovida a análise morfológica das ondas no traçado.

O eixo elétrico médio no plano frontal foi calculado diante do comportamento do complexo QRS na derivação bipolar I e unipolar aumentada aVF em relação à polaridade, determinando-se o menor QRS entre as duas unipolares aumentadas, aVR e Avl, como descrito por White II e Rhode (1974) e Fernandes et al. (2004). O escore cardíaco foi quantificado por meio da média aritmética do complexo QRS, incluindo as três derivações bipolares, I, II e III.

Os resultados do peso, da frequência cardíaca, da amplitude e duração dos complexos, do QTc, do desnivelamento ST, do eixo elétrico e escore cardíaco foram analisados por comparação de médias entre machos e fêmeas, utilizando-se o teste exato de Fischer, e o programa estatístico SigmaStat, considerando-se $\mathrm{P}<0,05$.

\section{RESULTADOS E DISCUSSÃO}

As análises hematológica e hemogasométrica indicaram desidratação e desequilíbrio eletrolítico, com valores elevados de hematócrito e proteína plasmática, $52 \pm 6 \%$ e $8,9 \pm 0,7 \mathrm{~g} \backslash \mathrm{dL}$, respectivamente. A hemogasometria revelou, ainda, valores dos íons cloro, 94 $\pm 4,6 \mathrm{mmoL} / \mathrm{L}$ e cálcio ionizado, $1,36 \pm 0,20 \mathrm{mmoL} / \mathrm{L}$ próximos aos limites inferiores, confirmando, assim, os resultados obtidos por Wickler e Foss (2004). Estes animais desenvolveram exaustão em função do metabolismo oxidativo decorrente da degradação aeróbia de carboidratos e ácidos graxos livres segundo Boffi (2006).

Em relação ao ritmo cardíaco, houve predomínio de taquicardia sinusal (Fig. 1) em 12 animais (85,8\%), resultado já observado por Dumont et al. (2010) no pós-exercício de equinos PSA, finalistas de provas de enduro, considerada por esses autores como resposta fisiológica. Em um equino (7,1\%), verificou-se arritmia sinusal (Fig. 2) com a frequência cardíaca (FC) variando de 48 a 78bpm. Conforme Dumont et al. (2010), esse é um achado frequente durante a fase de recuperação pós-exercício e está associada à alteração no tônus vagal.
Em outro animal, foi observada a ocorrência de complexo atrial prematuro (CAP), com variação de FC entre 93 e $111 \mathrm{bpm}$ (Fig. 3), caracterizando, desta forma, um complexo supraventricular prematuro (CSVP). Resultado semelhante ocorreu no estudo de Buhl et al. (2010), que o consideraram fisiológico na fase de recuperação. Entretanto, Bowen (2010) mencionou que as contrações atriais prematuras estavam associadas ao baixo desempenho atlético em função de alterações eletrolíticas, nos fluidos corpóreos e no status ácido-base, além de hipoxemia, isquemia, endotoxemia ou doenças cardíacas pré-existentes. Kiryu et al. (1999), em cavalos de corrida, observaram que $30 \%$ dos animais com baixo desempenho apresentaram arritmias cardíacas, porém sem manifestações clínicas, ao passo que outros $10 \%$ apresentaram clínica significativa, com mais de duas despolarizações prematuras durante o exercício máximo. Para o animal deste estudo, seria indicado uma avaliação eletrocardiográfica de repouso e durante o exercício, visto que, apesar de o CSVP ter sido observado na fase de recuperação - o que poderia ser fisiológico ocorreram mais de 20 despolarizações prematuras, em 60 segundos de avaliação.

A onda $\mathrm{P}$ mostrou-se positiva em todos os traçados, sendo positiva única $(+)$ em $72 \%$ dos achados e positiva bífida $(++)$ em $28 \%$. A onda $\mathrm{P}$ tendeu a tornar-se única, sobrepondo-se à onda $\mathrm{T}$, semelhante ao observado por Dumont et al. (2010), e foi considerada por Fázio et al. (2003) como um achado fisiológico durante o exercício ou no pós-imediato.

Foi observada redução na duração da onda $\mathrm{P}$, do intervalo PR e do intervalo QT, alongamento discreto do QTc e permanência da duração do complexo QRS (Tab. 1) em comparação aos valores de Evans (1994) e Fernandes et al. (2004), para equinos adultos em repouso. Estes resultados confirmaram os já apresentados por Evans (1994) e Dumont et al. (2010), ao sugerirem que o exercício promovia alterações mínimas no complexo QRS e redução na duração da onda $\mathrm{P}$ e nos intervalos PR e QT. Estes valores foram semelhantes aos obtidos por Fázio et al. (2003), durante o treinamento e após a corrida. 


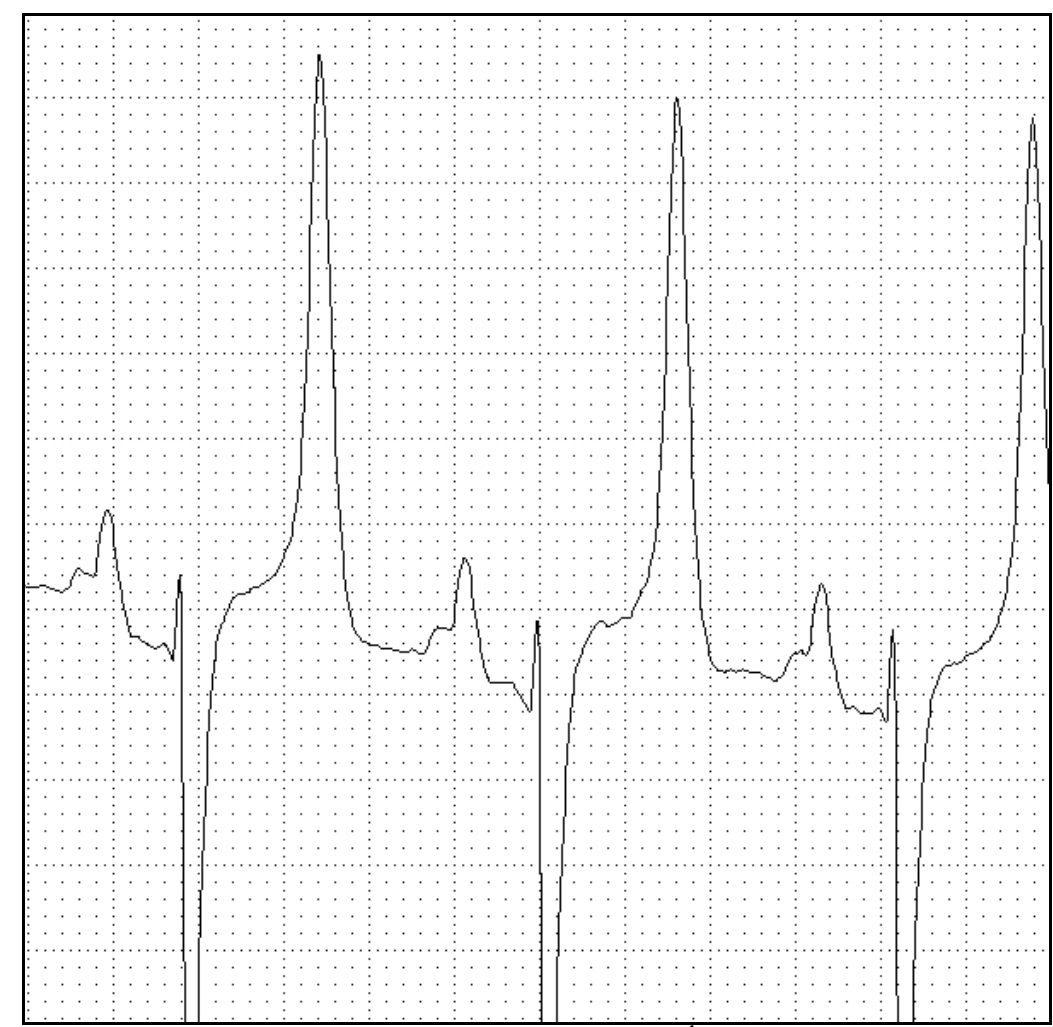

Figura 1. Traçado eletrocardiográfico de equino Puro Sangue Árabe na derivação bipolar II, em velocidade de $25 \mathrm{~mm} / \mathrm{s}$ apresentando taquicardia sinusal no pós-exercício imediato, com intervalo R-R de $1.725 \mathrm{~ms}$ e FC de $81 \mathrm{bpm}$.

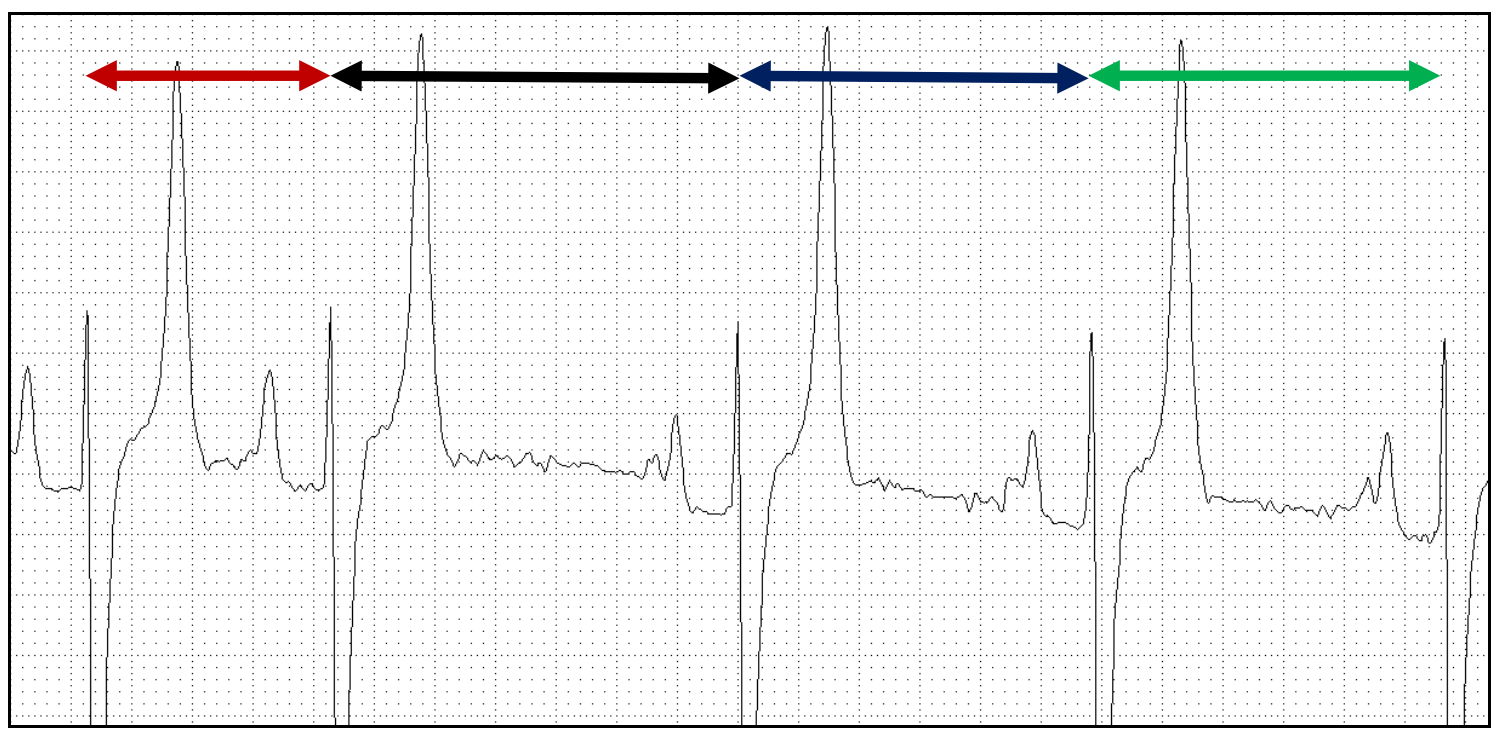

Figura 2. Traçado eletrocardiográfico de equino Puro Sangue Árabe na derivação bipolar II, em velocidade de $25 \mathrm{~mm} / \mathrm{s}$ apresentando arritmia sinusal no pós-exercício imediato. Pode-se observar a diferença na duração dos intervalos RR acima de 10\%. 


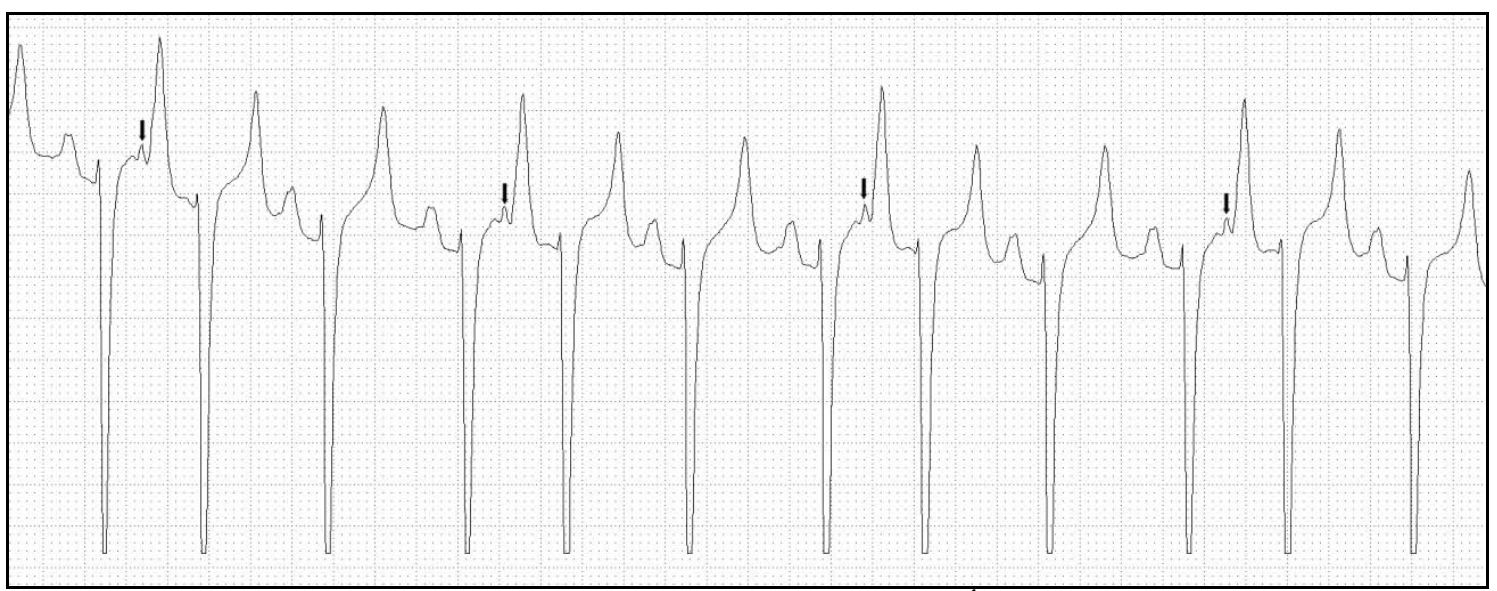

Figura 3. Traçado eletrocardiográfico de equino Puro Sangue Árabe na derivação bipolar II, em velocidade de $25 \mathrm{~mm} / \mathrm{s}$ apresentando complexo atrial prematuro com variação de FC entre 93 e $111 \mathrm{bpm}$ no pós-exercício imediato.

Tabela 1. Médias e erros-padrão das varáveis obtidas por meio de exame eletrocardiográfico de equinos Puro Sangue Árabe desclassificados em competições de enduro com longa duração

\begin{tabular}{|c|c|c|}
\hline \multirow{2}{*}{ Variável } & Macho & Fêmea \\
\hline & Média $\pm E P M$ & Média $\pm E P M$ \\
\hline peso (kg) & $393,17 \pm 9,38 *$ & $417,50 \pm 27,50 *$ \\
\hline FC(bpm) & $70,92 \pm 4,02$ & $73,50 \pm 7,50$ \\
\hline $\mathrm{P}(\mathrm{ms})$ & $129,00 \pm 7,81$ & $136,50 \pm 3,50$ \\
\hline $\mathrm{PR}(\mathrm{ms})$ & $224,75 \pm 7,67$ & $233,00 \pm 10,00$ \\
\hline QRS(ms) & $137,50 \pm 3,45$ & $136,50 \pm 3,50$ \\
\hline $\mathrm{QT}(\mathrm{ms})$ & $446,58 \pm 10,37$ & $448,00 \pm 5,00$ \\
\hline QTc(ms) & $474,83 \pm 5,86$ & $495,00 \pm 20,00$ \\
\hline $\mathrm{PU}(+)(\mathrm{mV})$ & $0,38 \pm 0,09$ & $0,72 \pm 0,22$ \\
\hline $\mathrm{R}(+)(\mathrm{mV})$ & $0,40 \pm 0,10$ & $0,10 \pm 0,02$ \\
\hline $\mathrm{S}(-)(\mathrm{mV})$ & $3,70 \pm 0,17$ & $2,23 \pm 0,55$ \\
\hline $\mathrm{ST}(+)(\mathrm{mV})$ & $0,31 \pm 0,04$ & $0,33 \pm 0,01$ \\
\hline $\mathrm{TU}(+)(\mathrm{mV})$ & $1,34 \pm 0,39$ & $1,84 \pm 0,98$ \\
\hline Escore cardíaco & $114,25 \pm 3,89$ & $111,00 \pm 5,00$ \\
\hline Eixo elétrico (-) & $105,00 \pm 7,83$ & $60,00 \pm 0,00$ \\
\hline
\end{tabular}

FC(bpm): frequência cardíaca, em batimentos por minuto; P(ms): duração da onda P, em milissegundos; PR(ms): duração do intervalo PR, em milissegundos; QRS(ms): duração do complexo QRS,em milissegundos; QT: duração do intervalo QT, em milissegundos; QTc: valor do índice QTc, intervalo QT corrigido pela FC, em milissegundos; R + (mv): amplitude da onda R positiva, em milivoltz; S - (mv): amplitude da onda S negativa, em milissegundos; ST + (mv): supradesnivelamento do segmento ST, em milivoltz; TU + (mv): amplitude da onda T única positiva, em milivoltz. *Na mesma linha, expressa diferença estatistica entre sexos, pelo teste exato de Fisher.

Quanto à morfologia, o complexo QRS comportou-se de duas formas distintas na derivação bipolar II. Em 50\% dos traçados observou-se a forma RS, e nos outros $50 \%$, a forma QRS, semelhante aos achados de Fernandes et al. (2004) e Dumont et al. (2010). O aumento da amplitude de $\mathrm{S}$ esteve relacionada com hipertrofia ventricular direita induzida pelo exercício, aspecto já descrito por Dumont et al. (2010).
Os cavalos em exaustão apresentaram aumento discreto no índice de Bazzet (QTc) (Tab. 1), indicando fadiga miocárdica moderada em resposta ao exercício realizado. Este índice, como sugerido por Sevestre (1982), está relacionado diretamente como estado de fadiga e pode atingir 550,0ms em casos de fadiga excessiva. A volta ao equilíbrio, com valor médio de 390,0ms, ocorreu no instante em que o animal se recuperava. Babusci e López (2006) 
consideraram impreciso esse índice e, sobretudo neste estudo, foi um meio auxiliar e de relevância na avaliação do desempenho do coração, em resposta ao esforço realizado.

Em seis animais (43\%) foi observado desnível do segmento ST acima de 0,3mv (Fig. 4). Essa variável, conforme sugerido por White II e Rhode (1974), poderia ser indicativo de choque, endotoxemia e dor abdominal, bem como de distúrbios eletrolíticos. De outra forma, em cavalos de enduro, a desidratação promoveria choque hipovolêmico, reduzindo substancialmente o volume e o tempo de diástole, assim como o suprimento sanguíneo e o fornecimento de oxigênio ao miocárdio, conforme verificou Muriel (2006). Tendo em vista que, ao instante da desclassificação, os animais já se encontravam com valores elevados de hematócrito e proteína plasmática, $52 \pm 6 \%$ e $8,9 \pm 0,7 \mathrm{~g} / \mathrm{dL}$, respectivamente, em função das perdas de fluidos, foi possível sugerir que a ocorrência de hipovolemia e desidratação justificariam o supradesnível do segmento ST.

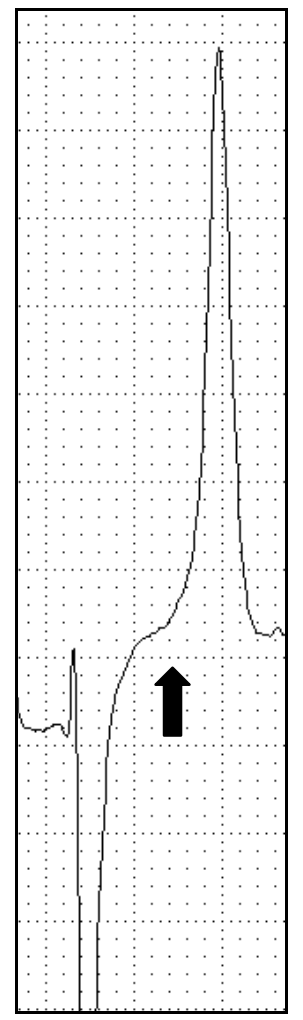

Figura 4. Traçado eletrocardiográfico de equino Puro Sangue Árabe demonstrando segmento ST horizontalizado, com supradesnível acima de $0,3 \mathrm{mV}$.
A onda $\mathrm{T}$ mostrou-se monofásica positiva em $72 \%$ dos animais e bifásica em $28 \%$, ou seja, sua morfologia foi extremamente variável, sobretudo não específica. Portanto, foi de pouca eficiência para um diagnóstico específico, pois esses arranjos poderiam ser observados também em doenças cardíacas, distúrbios eletrolíticos, miocardites e doenças sistêmicas, o que coincidiu com os achados de Dumont et al. (2010) e Trachsel et al. (2010), quando mencionaram que o estabelecimento de um padrão de onda $\mathrm{T}$ normal seria inespecífico e de difícil definição. Em função disso, Holmes e Rezakhani (1975) consideraram que a avaliação ideal de possíveis alterações deveria ser realizada por meio de monitoração do animal em repouso, durante o exercício e logo após a realização deste, objetivando, assim, determinar o comportamento individual dessa característica. Ainda, coincidindo com Holmes e Rezakhani (1975), a morfologia da onda T seria importante na identificação de hipóxia ou infarto do miocárdio, o que poderia ocorrer em atividades que produziriam desidratação extrema, em consequência da redução do aporte sanguíneo coronariano.

Foi observada, ainda, acentuada junção ST - T, que tornou o segmento ST mais horizontalizado em cinco animais (37,5\%). Em humanos, esse fato caracterizou-se como um achado típico de indivíduos com insuficiência coronariana crônica (Carneiro, 1997). Sobretudo na literatura consultada, não foram encontrados relatos específicos deste achado para equinos. Portanto, pode-se sugerir que essa alteração provavelmente foi induzida pelo exercício físico intenso, associado à desidratação e ao desequilíbrio eletrolítico a que estes animais estiveram expostos.

Verificou-se que o escore cardíaco médio (Tab. 1) foi condizente com os achados de Illera e Illera (1987), ao avaliarem cavalos de enduro de alto desempenho em treinamento, e de Dumont et al. (2010) durante competições. Assim, foi possível considerar que o exercício físico intenso resultou em elevada demanda do sistema cardiovascular. Ainda, Andrade et al. (2006) correlacionaram o escore cardíaco como um indicador da capacidade e do potencial atlético do animal. No entanto, nos animais mesmos que desclassificados, essa característica indicou aptidão atlética. 
O eixo elétrico expressou a condição cardíaca relacionada ao tipo de treinamento e à atividade a que os animais foram submetidos. $\mathrm{O}$ eixo apresentou desvio para a esquerda em cinco (36\%) animais, o que poderia indicar hipertrofia do ventrículo esquerdo; já o desvio para a direita, em nove (64\%) animais, caracterizou a ocorrência de hipertrofia do ventrículo direito, ambos condizentes, portanto, com treinamento, conforme White II e Rhode (1974), que denominaram este como hipertrofia cardíaca atlética. O desvio do eixo elétrico também esteve relacionado ao aumento de câmaras, como sugerido por Babusci e López (2006), em resposta adaptativa ao exercício submáximo de longa duração, assim como em resposta aos distúrbios eletrolíticos tais como os encontrados por White II e Rhode (1974) após competições de enduro de 50 a $100 \mathrm{~km}$, e essa rotação está associada à redução da concentração sérica de potássio, cloro e cálcio. De outra forma, esse eixo foi negativo em 14 animais, diferindo dos informes de Fernandes et al. (2004).

Em relação ao tratamento estatístico a que os parâmetros eletrocardiográficos foram submetidos, observou-se que não houve diferença significativa entre machos e fêmeas. Esse fato pode ter sido influenciado pelo pequeno número de fêmeas avaliadas ou, ainda, pela condição atlética similar entre os indivíduos dos dois gêneros.

\section{CONCLUSÃO}

Nos equinos desclassificados por exaustão, após terem participado de prova de enduro, foi possível a obtenção de traçados eletrocardiográficos de boa qualidade, verificando-se baixa incidência de artefatos e arritmias. O exame retratou, ainda, respostas adaptativas do coração frente aos efeitos do exercício de enduro, que foram expressas pelo alongamento do QTc - indicativo de fadiga miocárdica moderada em resposta ao exercício pelo supradesnível ST, em resposta à hipovolemia em função da desidratação induzida pela atividade - e pelo desvio à direita por parte do eixo elétrico no plano frontal - representando alterações eletrolíticas, aumento de câmara e hipertrofia secundárias ao treinamento.

\section{REFERÊNCIAS BIBLIOGRÁFICAS}

ANDRADE, A.F.C.; MICHIMA, L.E.S.; YONEZAWA, L.A. et al. Relação entre escore cardíaco e o condicionamento físico de equinos da raça Mangalarga. Rev. Bras. Cienc. Vet., v.13, p.125-130, 2006.

BABUSCI, M.; LÓPEZ, E.F. Sistema cardiovascular. In: BOFFI, F.M. (Ed). Fisiologia del ejercicio en equinos. Buenos Aires: InterMédica, 2006. p.61-85.

BOFFI, F.M. Princípios de entrenamiento. In: BOFFI, F.M. (Ed). Fisiologia del ejercicio en equinos. Buenos Aires: Inter-Médica, 2006. p.223-240.

BOWEN, I.M. Ambulatory electrocardiography and heart rate. In: MARR, C.M.; BOWEN, I.M. (Eds). Cardiology of the horse. London : Saunders, 2010. p.127-137.

BUHL, R.; MELDGAARD, C.; BARBESGAARD, L. Cardiac arrhythmias in clinical healthy showjumping horses. Eq. Vet. J., v.38, suppl., p.196-201, 2010.

CARNEIRO, E.F. O eletrocardiograma 10 anos depois. Rio de Janeiro: Enéas Ferreira Carneiro, 1997. 623p.

DUMONT, C.B.S.; LEITE, C.R.; MORAES, J.M. et al. Parâmetros eletrocardiográficos de equinos Puro Sangue Árabe submetidos a exercício prolongado de enduro. Cienc. Rural, v.40, p.1966-1973, 2010.

EVANS, D.L. The cardiovascular system: anatomy, physiology, and adaptations to exercise and training. In: ROSE, R.J.; HODGSON, D.R. (Eds). The Athletic horse. Philadelphia: Saunders, 1994. p.129-144.

FÁZIO, F.; FERRANTELLI, V.; PICCIONE, G. et al. Variations in some eletrocardiographic parameters in the trotter during racing and training. Vet. Res. v.27, suppl.1, p.229-232, 2003.

FERNANDES, W.R.; LARSSON, M.H.M.A. Alterações nas concentrações séricas de glicose, sódio, potássio, uréia e creatinina, em eqüinos submetidos a provas de enduro de $30 \mathrm{~km}$ com velocidade controlada. Cienc. Rural, v.30, p.393398, 2000. 
FERNANDES, W.R.; LARSSON, M.H.M.A.; ALVES, A.L.G. et al. Características eletrocardiográficas em equinos clinicamente normais da raça Puro Sangue Inglês. Arq. Bras. Med. Vet. Zootec., v.56, p.143-149, 2004.

GONDIM, F.J.; ZOPPI, C.C.; DOS REIS, L. et al. Possible relationship between performance and oxidative stress in endurance horses. $J$. Equine Vet. Sci. v.29, p.206-212, 2009.

HEIDBUCHEL, H.; HOOGSTEEN, J.; FAGARD, R. et al. High of right ventricular involvement in endurance athletes with ventricular arrhythmias. Eur. Heart J., v.24, p.1473-1480, 2003.

HINCHCLIF, K.W.; GEOR, R.J. Integrative physiology of exercise. In: HINCHCLIFF, K.W.; KANEPS, A.K.; GEOR, R.J. (Eds). Equine sports and medicine-basic and clinical sciences of the equine athlete. London: Saunders, 2004. p.3-9.

HOLMES, J.R.; REZAKHANI, A. Observations on the $\mathrm{T}$ wave of the equine eletrocardiogram. Equine Vet. J., v.1, p.55-62, 1975.

ILLERA, J.C.; ILLERA, M. Electrocardiography and heart score of horses competing in an endurance ride. Aust. Vet. J., v.64, p.88-89, 1987.

KIRYU, K.; MACHIDA, N.; KASHIDA, Y., et al. Pathologic and electrocardiographic findings in sudden cardiac death in racehorses. J. Vet. Med. Sci., v.61, p.921-928, 1999.

MURIEL, M.G. Transtornos hidroeletrolíticos. Patologias que afetam el rendimiento desportivo. In: BOFFI, F.M. (Ed). Fisiologia del ejercicio en equinos. Buenos Aires: Inter-Médica, 2006. p.282-286.
PETER, E.M. Nutricional aspects in ultraendurance exercise. Curr. Opinion Clin. Nutr. Metab. Care, v.6, p.427-434, 2003.

PETSCHE, V.M.; DERKSEN, F.J.; BERNIE, C.E. et al. Effect of head position on upper airway function in exercising horses. Equine Vet. J., v.18, suppl., p.18-22, 1995.

SEVESTRE, J. A eletrocardiografia no cavalo. Hora Vet., n.10, p.28-36, 1982

SERRANO, A.L.; QUIROZ-ROTHE, E.; RIVERO, J.L. Early and long-term changes of equine skeletal muscle in response to endurance training and detraining. Eur. J. Physiol., v.441, p.263-274, 2000.

TRACHSEL, D.S.; BITSCHNAU, N.; WALDERN, M.A. Observer agreement for detection of cardiac arrhythmias on telemetric ECG recordings obtained at rest, during and after exercise in Warmblood horses. Equine Vet. J., v.38, suppl., p.208-215, 2010.

WHITE II, N.A.; RHODE, E.A. Correlation of electrocardiographic findings to clinical disease in the horse. J. Am. Vet. Med. Assoc., v.164, p.46-56, 1974.

WICKLER, S.J.; FOSS, M.A. Veterinary aspects of endurance riding. In: HINCHCLIFF, K.W.; KANEPS, A.K.; GEOR, R.J. (Eds). Equine sports and medicine-basic and clinical sciences of the equine athlete. London: Saunders, 2004. p.1105-1117. 